\title{
ASYMPTOTIC RESULTS FOR THE SOLUTIONS OF A CERTAIN DIFFERENTIAL EQUATION
}

\author{
J. J. MAHONY
}

(Received 3 October 1969)

Communicated by A. F. Pillow

\section{Introduction}

The asymptotic behaviour, for large real positive values of the independent variable $t$, of solutions of the ordinary differential equation

$$
\ddot{y}+t^{-1} y \sin a t=0
$$

is considered. (The dots denote differentiation with respect to $t$.) It is shown that this asymptotic behaviour can be obtained by comparison with the differential equation

$$
\ddot{y}+y\left(2 a^{2} t^{2}\right)^{-1}=0 .
$$

For both equations when $a^{2}<2$ the solutions are unbounded oscillatory functions but when $a^{2}>2$ the solutions are essentially monotone. The critical case $a^{2}=2$ has not been investigated here although the present results suggest that the solutions are probably monotone. The asymptotic estimates of the solutions of equation (1) are first obtained by heuristic means commonly used by Applied Mathematicians working on singular perturbation problems. A basis for a formal expansion is obtained in terms of a recurrence relation and the first few terms are displayed explicitly. These formal expansion estimates are used to guide a rigorous analysis which is essentially an extension of the techniques used in validating solutions obtained by the Langer related equation technique. For the oscillatory case, a pair of Volterra integral equations is used to justify the asymptotic representations for an arbitrary large interval for $t$ but not including the point at infinity.

For the monotone case, a pair of Fredholm integral equations are used to justify the asymptotic representations in a neighbourhood of infinity. A brief discussion is given as to how the arguments can be applied to other powers than $t^{-1}$ and to more general periodic functions than sin at that occur in equation (1). 


\section{Heuristic and formal determination of asymptotic behaviour}

The question of determining whether solutions of differential equations, such as

$$
\ddot{y}+t^{-1} y \sin t=0,
$$

had oscillatory or monotone solutions for $t$ real large positive was brought to the author s attention by Dr. J. Wong. For the inferential methods often used in settling such questions it is convenient to have an informed guess as to the answer so as to direct the line of the argument. The above equation seemed rather a sensitive case and the author tried using the method of multiple scales (vide Cole (1)) in a somewhat unusual fashion. This method is used when there are two interacting scales of action differing by a small parametric factor. The analysis revealed that the more general equation (1) could be studied by the same means and that there was a startling dichotomy for $a^{2}$ greater than or less than 2. It may be noted that a scale change of $t$ introduces a factor multiplying the coefficient of $y$ so that equation (1) may be regarded as a reduced form for a broader class of equations.

In the present problem the variable $t^{-1}$ can be used as a measure of smallness to replace the small numerical parameter on which the method of multiple scales is worked. The differential equation (1) implies that $t$ also has a role in which the scale is set by $\sin a t$ and this must be reflected in the solution.

But since for large $t, t^{-1} \sin a t$ has a small mean value there will also be a longer scale over which $\ddot{y}$ and the mean of $y t^{-1} \sin a t$ strike some form of balance. The first step is to determine the scale of this balance. If the formally smaller order term is neglected the differential equation has as its first approximation $\ddot{y}=0$. Because the differential equation is linear the scale of $y$ is unimportant so that the first approximation is $y_{0}(\tau)$ where $\tau$ is the unknown slow time scale and $y_{0}$ also remains undetermined. An improvement of this follows from the first iterate

$$
\ddot{y}+y_{0}(\tau) t^{-1} \sin a t=0 .
$$

Integration of this and approximating the integrals involved, while maintaining $\tau$ constant, suggest that

$$
y=y_{0}(\tau)+y_{0}(\tau)\left(a^{2} t\right)^{-1} \sin a t
$$

is a better approximation. At this stage no balance on the scale of $\tau$ has been needed to keep the approximation appearing satisfactory. However the next stage the approximation becomes

$$
\ddot{y}+\left\{y_{0}+y_{0}\left(a^{2} t\right)^{-1} \sin a t\right\} t^{-1} \sin a t=0
$$

and for the first time the integration of a trigonometric function of non-zero mean occurs. If this mean were accepted and the $\tau$ scale still be considered unimportant $y$ would grow as $\ln t$ and the perturbation method fail. It must be then that the 
scale of $\tau$ is such as to balance this mean which is $\frac{1}{2} y_{0}(a t)^{-2}$. The work of Fowkes (2) has shown the way to cope with such a term is to re-order the calculation procedure by rewriting the equation (1) as

$$
L(y)=\ddot{y}+\frac{1}{2}(a t)^{-2} y=\left\{\frac{1}{2}(a t)^{-2}-t^{-1} \sin a t\right\} y
$$

and now to dominate the equation by the operator on the left hand side. Fowkes' work, in a different context, suggests that the above form is the way to apply the Langer related equation technique to the differential equation (1).

The solutions of the dominating equation $L(y)=0$ take the form $t^{\lambda}$ where $\lambda$ is either root of

$$
\lambda^{2}-\lambda+\frac{1}{2} a^{-2}=0
$$

When $a^{2}>2$ the roots are real, distinct and lie in the interval $(0,1)$ so that solutions of the dominating equation are monotone. The sum of the roots is unity but in this section $m$ will be used to denote either rather than take advantage of this special property. When $a^{2}<2$ the roots of equation (4) are conjugate complex and the nature of the solutions is most clearly conveyed by writing the solutions of the dominating equation in the form

$$
t^{\frac{1}{2}} \cos (\beta \ln t) \text { and } t^{\frac{1}{2}} \sin (\beta \ln t) \text { where } \beta=\frac{1}{2}\left\{2 a^{-2}-1\right\}^{\frac{1}{2}} \text {. }
$$

in this case the dominating equation possesses solutions which are both unbounded and oscillatory and the slow scale in $t$ is apparent. For the monotone case there is no slow scale involved.

Formal solutions can now be obtained by substitution of expansions suggested by the above considerations. When the solutions are expected to be monotone (i.e. $a^{2}>2$ ) one tries expansions of the form

$$
y \sim \sum_{r=0}^{\infty} t^{m-r} A_{r}(t)
$$

wherein it is to be required that the functions $A_{r}(t)$ should be uniformly bounded for large values of $t$. Substitution of this into equation (1) and the equating of the coefficients of powers of $t$ to zero lead to the set of equations

$$
\begin{aligned}
& \ddot{A}_{0}=0 \quad \ddot{A}_{1}=-\sin a t \\
& \ddot{A}_{r+2}+2(m-r-1) \dot{A}_{r+1}+(m-r)(m-r-1) A_{r}+A_{r+1} \sin a t=0, \quad r \geqq 0 .
\end{aligned}
$$

Without loss of generality the solution of the first equation may be taken as $A_{0}=1$ and then the second equation has bounded solutions of the form

$$
A_{1}=a^{-2} \sin a t+C_{1}
$$

where $C_{1}$ is an as yet undetermined constant. When the equation for determining $A_{2}$ is considered the only trigonometric term which leads to unbounded terms in $A_{2}$ is $A_{1} \sin a t$. This has a mean value $\frac{1}{2} a^{-2}$ but there are additional constant 
terms, which come from $A_{0}$, leading to a total mean $\left(m^{2}-m+\frac{1}{2} a^{-2}\right)$ and this vanishes by equation (4). This is not accidental but a demonstration of the appropriate choice of dominating equation. The remaining terms in the equation determining $A_{2}$ yield

$$
A_{2}=2(m-1) a^{-3} \cos a t-(2 a)^{-3} \cos 2 a t+C_{2}
$$

where $C_{2}$ along with $C_{1}$ remains an undetermined constant. In the equation for $A_{3}, A_{2} \sin$ at has zero means so that $A_{3}$ will remain bounded provided $C_{1}$ is chosen to be zero. The solution for $A_{3}$ involves only sine and no cosine functions. The equation for $A_{4}$ thus has a contribution to the mean from $A_{3} \sin a t$ but there is an available the constant $C_{2}$ which can be chosen so that $A_{4}$ is bounded. It is now apparent that odd numbered coefficients consist solely of sines, the even numbered of cosines plus a constant of integration which is determined so that a later coefficient will remain bounded. The above formal procedure can be continued in this pattern ad infinitum.

For the oscillatory case the form of solutions of the dominating equation suggests attempting a formal asymptotic expression,

$$
y=t^{\frac{1}{2}} \sum_{r=0}^{\infty} t^{-r}\left\{A_{r}(t) \cos (\beta \ln t)+B_{r}(t) \sin (\beta \ln t)\right\}
$$

wherein, as before, the $A_{r}$ and $B_{r}$ are to be required to be uniformly bounded for large values of $t$ and also not to be slowly varying as is $\cos (\beta \ln t)$. With this case the calculation difficulties are greater than for the monotone case. The same type of general arguments can be applied so that the function $A_{n}$ and $B_{n}$ and these arguments may be necessary for some of the more general equations considered in the final section of this paper. However in the present context we can take advantage of the calculations for the monotone case by taking real and imaginary parts of the complex-valued solution of equation (6) arising because $m$ is complex. One solution has the first two terms given by

$$
y=t^{\frac{1}{2}}\left\{\cos (\beta \ln t)+a^{-2} t^{-1} \sin (\beta \ln t) \sin a t\right\}+0\left(t^{-\frac{3}{2}}\right)
$$

For guidance in later work it is useful to note that the largest term in $y$ is slowly varying. Further the largest term in $\dot{y}$, though smaller by $0\left(t^{-1}\right)$ than $y$, contains both slowly and rapidly varying functions. It is the derivative of these rapidly varying functions which makes $\ddot{y}$ the same order as $\dot{y}$ and which permit the balance to be struck between $\ddot{y}$ and $t^{-1} y \sin a t$.

\section{Proof of the asymptotic nature of the oscillatory solution}

The basis of the proof to be given is really an adaption of the Langer related equation technique. Because the solutions are unbounded and oscillatory it does not appear feasible to establish the validity of the asymptotic representation in the 
neighbourhood of infinity. Such proof requires precise evaluation of the phase and mathematical tools to cope with such a problem are sadly lacking. Thus one seems forced to consider behaviour of the representation in an interval $\left(T, T^{\prime}\right)$ where $T$ is to be chosen sufficiently large and one tries to make $T^{\prime}$, which depends on $T$, as large as possible and certainly large enough for the slow oscillations to bave been demonstrated as being a real phenomenon. As is standard practice in the Langer related equation theory the differential equation is written in the form

$$
\ddot{y}+\left(2 a^{2} t^{2}\right)^{-1} y=\left\{\left(2 a^{2} t^{2}\right)^{-1}-t^{-1} \sin a t\right\} y
$$

and converted into an integral equation. For reasons given above it is assumed that the problem is to be treated as an initial value problem with initial data given at $t=T$. It follows, by the method of variation of parameters, that the appropriate integral equation is.

$$
\begin{array}{r}
y=t^{\frac{1}{2}}\{A \cos (\beta \ln t)+B \sin (\beta \ln t)\}+\beta^{-1} \int_{T}^{t}\left\{\left(2 a^{2} s\right)^{-1}-\sin a s\right\} y(s)(t / s)^{\frac{1}{2}} \\
\sin [\beta \ln (t / s)] d s
\end{array}
$$

where $A$ and $B$ are constants specifying a particular solution. It is convenient to introduce a new variable defined by

$$
y=t^{\frac{1}{2}} Y(t)
$$

so that $Y$ satisfies

$$
\begin{aligned}
Y= & A \cos (\beta \ln t)+B \sin (\beta \ln t) \\
& +\beta^{-1} \int_{T}^{t}\left\{\left(2 a^{2} s\right)^{-1}-\sin a s\right\} Y(s) \sin [\beta \ln (t / s)] d s .
\end{aligned}
$$

As it stands iteration of this Volterra integral equation will give useful results only for $(t-T) \ln (t / T)$ small and this is an insufficient range. However a more useful form can be obtained by integrating by parts. If the more rapidly varying $\sin a s$ is integrated and the more slowly varying part $Y(s) \sin [\beta \ln (t / s)]$ is differentiated the integrand will be reduced by a factor $O\left(s^{-1}\right)$. It is possible to include the contribution from $t=T$ within the general constants $A$ and $B$. The modified equation thus obtained is

$$
\begin{aligned}
Y(t)= & A \cos (\beta \ln t)+B \sin (\beta \ln t)+\beta^{-1} \int_{T}^{t}\left(2 a^{2} s\right)^{-1} Y(s) \sin [\beta \ln (t / s)] d s \\
& -(\alpha \beta)^{-1} \int_{T}^{t} \cos a s\left\{\dot{Y}(s) \sin [\beta \ln (t / s)]-\beta s^{-1} Y(0) \cos [\beta \ln (t / s)]\right\} d s .
\end{aligned}
$$

While this is no longer an integral equation the formal estimates of section 2 agree with the first iterate of the right hand side. Moreover the iterate should be useful as long as $(t-T) / T$ is small. But further improvement is necessary if one is to cover an interval containing many zeros of $\cos (\beta \ln t)$. The second integral may be integrated by parts to yield 
$Y(t)\left\{1-a^{2} t^{-1} \sin a t\right\}=A \cos (\beta \ln t)+B \sin (\beta \ln t)$

$$
\begin{aligned}
& +\beta^{-1} \int_{T}^{t}\left(2 a^{2} s\right)^{-1} Y(s) \sin [\beta \ln (t / s)] d s \\
& +\left(a^{-2} \beta^{-1}\right) \int_{T}^{t} \sin a s\left\{\left[\ddot{Y}-\beta^{2} s^{-2} Y(s)\right] \sin [\beta \ln (t / s)]\right. \\
& \left.+\left[\beta s^{-2} Y(s)-2 \beta s^{-1} \dot{Y}(s)\right] \cos [\beta \ln (t / s)]\right\} d s .
\end{aligned}
$$

The only term in the second integral which is not $O\left(s^{-2}\right)$ is the term involving $\ddot{Y}$. If $\ddot{Y}$ is eliminated using the differential equation (1) and the definition $Y$ in equation (11) the only term which is not $0\left(s^{-2}\right)$ is $-a^{2} \beta^{-1} s^{-1} Y \sin ^{2} a s$. The more rapidly oscillitary part has a non-zero mean which can be derived through $\sin ^{2}$ as $=$ $\frac{1}{2}(1-\cos 2 a s)$. It is immediately apparent that the first integral in equation (12) is cancelled by the contribution of the mean. This should not be surprising because the above calculations are really only a development of the heuristic arguments which led to the dominating equation. There remains however one term in the integrand, namely $\frac{1}{2} a^{2} \beta^{-1} s^{-1} Y \cos 2 a s \sin [\beta \ln t / s]$ which is not as small as $s^{-2}$ but this may be removed by a further integration by parts. The resulting equation after all these calculations is

$$
\begin{aligned}
Y(t)\left\{1-\left(a^{2} t\right)^{-1} \sin a t\right\}= & A \cos (\beta \ln t)+B \sin (\beta \ln t) \\
& +\int_{T}^{t} s^{-2} Y(s) K_{1}(s, t) d s+\int_{T}^{t} s^{-1} \dot{Y}(s) K_{2}(s, t) d s
\end{aligned}
$$

where $K_{1}$ and $K_{2}$ are the uniformly continuous functions

$$
\begin{aligned}
K_{1}(s, t)= & a^{-2} \beta^{-1} \sin a s\left\{\beta \cos [\beta \ln (t / s)]-\left(\beta^{2}-\frac{1}{4}\right) \sin [\beta \ln (t / s)]\right\} \\
& +\frac{1}{4} a^{-3} \beta^{-1} \sin 2 a s\{\sin [\beta \ln (t / s)]+\beta \cos [\beta \ln (t / s)]\} \quad \text { and } \\
K_{2}(s, t)= & -a^{-2} \beta^{-1} \sin a s\{\beta \cos [\beta \ln (t / s)]+\sin [\beta \ln (t / s)]\} \\
& +\frac{1}{4} a^{-3} \beta^{-1} \sin 2 a s \sin [\beta \ln (t / s)] .
\end{aligned}
$$

The formal expansions suggest that $\dot{Y}$ is $0\left(t^{-1}\right)$ so that a new variable $Z$, expected to be of unit order, is introduced by

$$
\dot{Y}=t^{-1} Z \text {. }
$$

Differentiation of equation (13) together with some rearrangement leads to the equation

$$
\begin{aligned}
Z(t)-Y(t) a \cos a t= & -\beta A \sin (\beta \ln t)+\beta B \cos (\beta \ln t) \\
& +\int_{T}^{t} s^{-2} Y(s) K_{3}(s, t) d s+\int_{T}^{t} s^{-2} Z(s) K_{4}(s, t) d s
\end{aligned}
$$

where

$$
K_{3}(0, t)=t K_{1 t}(s, t) \text { and } K_{4}(s, t)=t K_{2 t}(s, t)
$$


and these are uniformly bounded, continuous for large values of $t$. Together with the modified form of equation (13)

$$
\begin{aligned}
Y\left\{1-a^{-2} t^{-1} \sin a t\right\}= & A \cos (\beta \ln t)+B \sin (\beta \ln t) \\
& +\int_{T}^{t} s^{-2} Y(s) K_{1}(s, t) d s+\int_{T}^{t} s^{-2} Z(s) K_{2}(s, t) d s
\end{aligned}
$$

this forms a pair of coupled Volterra integral equations for $Y$ and $Z$. By restricting $t$ to a range in which $T^{-2}(t-T)$ is suitably small it is possible to obtain bounds on the maximum values of $Y$ and $Z$ in the interval $(T, t)$ for in this range the integrals contribute only small multiples of $\operatorname{Max} Y$ and $\operatorname{Max} Z$. The resulting bounds being independent of $T$, then permits equations (16b) to be interpreted as

$$
Y=\{A \cos (\beta \ln t)+B \sin (\beta \ln 1)\}\left\{1-a^{-2} t^{-1} \sin a t\right\}^{-1}+O\left(\frac{t-T}{T^{2}}\right)
$$

so that the leading term provides an asymptotic representation for $Y$ in an interval $\left(T, T+T^{\mu}\right)$ where $0<\mu<2$. Further higher order expansions are available by iterating the coupled integral equations before performing the inequality arguments. It should be noted that the additional terms do not extend the range over which the asymptotic representation is valid but merely increase the accuracy of the representation. The limit on the domain of validity arises from the need to establish bounds on $Y$ and $Z$ and in essence this hinges crucially on the argument

$$
|Y| \gg\left|\int_{T}^{t} s^{-2} Y(s) K_{1}(s, t) d s\right|
$$

where $Y$ is an unknown function and this places an overall restriction on the extent of the range. The estimates of the various terms are rather crude and probably the representation is valid over a larger range of $t$ but there appears to be no way of establishing this.

\section{Validity of the expansion for the monotone case}

In the case of slowly oscillating solutions it is acceptable to have a representation valid over an arbitrarily large number of oscillations even if it does not include the point at infinity. Such a localized solution for the apparently monotone case would not be acceptable for the solutions might be oscillatory on a longer time scale. Merely repeating a discussion similar to the oscillating case is therefore inadequate. Thus once monotone solutions are suspected it appears essential to establish the validity of the asymptotic representation in a neighbourhood of the point at infinity. However, this presents much greater difficulties because for the initial value problem considered in the previous section there are known existence theorems. For a neighbourhood of infinity, existence of solutions has not been 
established for equation (1). It will therefore be necessary to establish the existence of solutions of the required form as well as to show that the series are valid approximations to the actual solutions.

There will be further technical difficulties in using an integral equation approach because of the uncertainty that the integrals involved converge. The dominating equation has solutions $t^{m}$ and $t^{n}$ where $m$ and $n$ are defined so that $0<m<n<1$. In an endeavour to minimize these technical difficulties it was decided to seek the solution for which $t^{m}$ was the leading term in the asymptotic representation. Again equation (9) is solved by variation of parameters and it is found that if one wishes the integrals involved to be convergent (assuming the behaviour of the formal representation applies) the boundary conditions must be applied in such a way as to lead to the Fredholm integral equation

$$
\begin{aligned}
y= & t^{m}-(n-m)^{-1}\left\{t^{n} \int_{t}^{\infty}\left[\left(2 a^{2} s\right)^{-1}-\sin a s\right] y s^{-n} d s\right. \\
& \left.+t^{m} \int_{T}^{t}\left[\left(2 a^{2} s\right)^{-1}-\sin a s\right] y(s) s^{-m} d s\right\}
\end{aligned}
$$

the $t^{n}$ term disappears in the limit $t$ tending to infinity and the first integral is convergent assuming $y(s)$ is $O\left(s^{m}\right)$. A procedure similar to that used in section 3, may be applied to reduce systematically the size of the integrands. Again the terms $\left(2 a^{2} s\right)^{-1}$ disappear, being cancelled by the mean of the $\sin ^{2}$ as which arises after two integration by parts. After extensive calculations one obtains

(18) $y(t) h(t)=t^{m}+\int_{T}^{\infty} s^{-2} y(s) L_{1}(s, t) d s+\int_{T}^{\infty} s^{-1} \dot{y}(s) L_{2}(s, t) d s$

where

$$
L_{1}(s, t)=(n-m)^{-1}\left\{\begin{array}{l}
{\left[\sin a s \cdot n(n+1) a^{-2}+\frac{1}{4} a^{-3}(n+1) \sin 2 a s\right](t / s)^{n} \quad s>t} \\
{\left[m(m+1) a^{-2} \sin a s+\frac{1}{4} a^{-3}(m+1) \sin 2 a s\right](s / t)^{m} s<t}
\end{array}\right.
$$

and

$$
L_{2}(s, t)=-(n-m)^{-2} \begin{cases}{\left[2 n a^{-2} \sin a s+\frac{1}{4} a^{-3} \sin 2 a s\right](t / s)^{n}} & s>t \\ {\left[2 m a^{2} \sin a s+\frac{1}{4} a^{-3} \sin 2 a s\right](s / t)^{m}} & s<t\end{cases}
$$

and

(19c) $h(t)=\left\{1-a^{-2} t^{-1} \sin a t\right)$.

The kernels $L_{1}$ and $L_{2}$ are bounded, independent of $t$ in $(T, \infty)$. Again it is necessary to construct a second integral relation which can be obtained by differentiating equation (18) with respect to $t$. The result may be written in the form

$$
\dot{y} g_{1}(t)=y t^{-1} g_{2}(t)+m t^{m-1}+\int_{T}^{\infty} s^{-3} y(s) L_{3}(s, t) d s+\int_{T}^{\infty} s^{-2} \dot{y}(s) L_{4}(s, t) d s
$$

where

$$
g_{1}(t)=1+a^{-2} t^{-1} \sin a t+a^{-3} t^{-1} \sin 2 a t .
$$


(21b)

$$
g_{2}(t)=a^{-1} \cos a t+(m+n) a^{-2} t^{-1} \sin a t+a^{-3} t^{-2} \sin a t
$$

and

$$
\begin{aligned}
& L_{3}(s, t) \\
& =(n-m)^{-1}\left\{\begin{array}{ll}
n\left[n(n+1) a^{-2} \sin a s+\frac{1}{4}(n+1) a^{-3} \sin 2 a s\right](t / s)^{n-1}, & s>t \\
-m\left[m(m+1) a^{-2} \sin a s+\frac{1}{4}(m+1) a^{-3} \sin 2 a s\right](s / t)^{m-1}, & s<t
\end{array}\right\} \\
& L_{4}(s, t)=-(n-m)^{-1}\left\{\begin{array}{ll}
n\left[2 n a^{-2} \sin a s+\frac{1}{4} a^{-3} \sin 2 a s\right](t / s)^{n-1}, & s>t \\
-m\left[2 m a^{-2} \sin a s+\frac{1}{4} a^{-3} \sin 2 a s\right](s / t)^{m-1}, & s<t
\end{array}\right\} .
\end{aligned}
$$

Again the kernels $L_{3}$ and $L_{4}$ are bounded, independent of both $t$ and $T$. Consider the iterative scheme

$$
\begin{aligned}
& y_{0}(t) h(t)=t^{m} \\
& \dot{y}_{0}(t) g_{1}(t)=y_{0}(t) t^{-1} g_{2}(t)+m t^{m-1}
\end{aligned}
$$

and for

$$
\begin{aligned}
& r \geqq 0 y_{r+1}(t) h(t)=\int_{T}^{\infty} s^{-2} y_{r}(s) L_{1}(s, t) d s+\int_{T}^{\infty} s^{-1} \dot{y}(s) L_{2}(s, t) d s \\
& \dot{y}_{r+1} g_{1}(t)=y_{r+1} t^{-1} g_{2}(t)+\int_{T}^{\infty} s^{-3} y_{r}(s) L_{3}(s, t) d s+\int_{T}^{\infty} s^{-2} y_{r}(s) L_{4}(s, t) d s .
\end{aligned}
$$

For sufficiently large values of $T$ such that both $h$ and $g_{1}$ are greater than $\frac{1}{2}$, there exists a constant $B_{0}$ such that

$$
\begin{aligned}
& \left|y_{0}(t)\right|<2 t^{m-1} \\
& \left|\dot{y}_{0}(t)\right|<B_{0} t^{m-1}
\end{aligned}
$$

It is therefore possible to show inductively

$$
\begin{aligned}
& \left|y_{r}(t)\right|<A_{r} T^{m-r} \\
& \left|\dot{y}_{r}(t)\right|<B_{r} T^{m-r-1}
\end{aligned}
$$

where

$$
\begin{aligned}
& A_{r}<[\operatorname{Max}\{1 / h(t)\}]\left\{\left[\operatorname{Max} L_{1}\right] A_{r-1}+\left[\operatorname{Max} L_{2}\right] B_{r-1}\right\} \\
& B_{r}<\left[\operatorname{Max}\left\{1 / g_{1}(t)\right\}\right]\left\{\left[\operatorname{Max} g_{2}\right] A_{r}+\left[\operatorname{Max} L_{3}\right] A_{r-1}+\left[\operatorname{Max} L_{4}\right] B_{r-1}\right\},
\end{aligned}
$$

and one can eliminate $A_{r}$ from the equation for $B_{r}$ using the first equation of this pair. It is thus possible to show that there exists an $M$ such that

$$
A_{r}+B_{r}<M\left(A_{r-1}+B_{r-1}\right)
$$

and hence that both $A_{r}$ and $B_{r}$ are less than $C M^{r}$ for some constant $C$. It thus follows that

$$
\sum_{r=0}^{\infty} y_{r}(t) \text { and } \sum_{r=0}^{\infty} y_{r}(t)
$$


are uniformly convergent in $[T, \infty)$ provided $T>(1 / M)$ and also meets all the earlier constraints. The iteration therefore converges to a function pair $y(t)$, $\dot{y}(t)$ and the function $y(t)$ may be shown to satisfy the differential equation (1). Further equations (22) imply that $\sum_{r=0}^{N} y_{r}$ is an asymptotic representation of this solution for large enough $T$. The monotone nature of one solution is thus established and the standard theorems on interlacing of zero simply that any other solution is monotone. If we denote the above solution by $Z$ then a further solution obtained by reduction of order is,

$$
y=z \int^{t}[z(s)]^{-2} d s .
$$

To demonstrate the asymptotic behaviour of this solution one notes that

and hence

$$
[z(s)]^{-2}=s^{-2 m}+O\left(s^{-1-2 m}\right)
$$

$$
\begin{aligned}
y & =-z t^{(2 m-1)} /(2 m-1)+O(1) \\
& =-(2 m-1)^{-1} t^{1-m}+O(1)
\end{aligned}
$$

and in essence this is the leading term for the formal asymptotic expansion of the larger solution, thus verifying to leading order the formal series. The formal expansions were uniquely determined by the requirements of having the given form and satisfying the differential equation. Thus the formal series expansion must be a valid asymptotic representation since it takes the same form as the result of iterating the integral equation.

\section{Some extensions of the analysis}

The methods employed in this paper are applicable to a much broader class of problems than merely the original differential equation (1). It is a trivial extension to apply the method to any equation of the form

$$
\ddot{y}+\left\{t^{-1} \sin a t+\sum_{r=2}^{\infty} b_{r} t^{-r}\right\} y=0 .
$$

Obviously the solutions are monotone provided $4\left\{b_{2}+\left(2 a^{2}\right)^{-1}\right\}$ is less than one and oscillatory if it is greater than one. The same type of arguments may be applied to the equation

$$
\ddot{y}+y t^{-k} \sin a t=0
$$

where $0<k<1$, the dominating equation is

$$
\ddot{y}+\left(2 a^{2}\right)^{-1} t^{-2 k} y=0
$$

and the asymptotic behaviour of the solution is given by

$$
y \sim t^{k / 2} \exp \left\{i 2^{-\frac{1}{2}} a^{-1}(1-k)^{-1} t^{1-k}\right\}
$$


so that the solutions are unbounded and oscillatory. When $k$ is greater than one the solutions are monotone since the comparison differential equation (23) has infinity as a regular point

Perhaps the most interesting extension of equation (1) is

$$
\ddot{y}+t^{-1} p(t) y=0
$$

where $p(t)$ is a periodic function of zero mean and period $\tau$. Let $p(t)$ be represented by its Fourier series

$$
p(t)=\sum_{r=1}^{\infty}\left\{a_{r} \cos (2 r \pi t / \tau)+b_{r} \sin (2 \pi r t / \tau)\right\}
$$

In repeating the argument of section 2 an appropriate two-fold integral of $p(t)$ is

$$
-(\tau / 2 \pi)^{2} \sum_{r=1}^{\infty} r^{-2}\left\{a_{r} \cos (2 \pi r t / \tau)+b_{r} \sin (2 \pi r t / \tau)\right\}
$$

and the coefficient, which is obtained by averaging the product $p(t)$ with this integral, of the comparison equation is given by

$$
\tau^{2} /\left(8 \pi^{2}\right) \sum_{r=1}^{\infty} r^{-2}\left(a_{r}^{2}+b_{r}^{2}\right)
$$

This sum can be converted in terms of integrals of $p(t)$ as follows

$$
\int_{0}^{t} p(s) d s=\left(\frac{\tau}{2 \pi}\right) \sum_{r=1}^{\infty} r^{-1}\left\{a_{r} \sin (2 \pi r t / \tau)-b_{r} \cos (2 \pi r t / \tau)+b_{r}\right\} .
$$

Now $\sum b_{r} / r$ is the mean value of $\int_{0}^{t} p(s) d s(2 \pi / \tau)$ over the period $\tau$ so that

$$
\begin{aligned}
\sum_{r=1}^{\infty} b_{r} / r & =2 \pi \tau^{-2} \int_{0}^{\tau} \int_{0}^{t} p(s) d s d t \\
& =2 \pi \tau^{-2} \int_{0}^{\tau}(\tau-s) p(s) d s \\
& =-2 \pi \tau^{-2} \int_{0}^{\tau} s p(s) d s
\end{aligned}
$$

since $p$ has zero mean. Hence

$$
\int_{0}^{t} p(s) d s+\tau^{-1} \int_{0}^{\tau} s p(s) d s=\left(\frac{\tau}{2 \pi}\right) \sum_{r=1}^{\infty} r^{-1}\left\{a_{r} \sin (2 \pi r t / \tau)-b_{r} \cos (2 \pi r t / \tau)\right\}
$$

If one introduces the notation

$$
\alpha=\tau^{-1} \int_{0}^{\tau} s p(s) d s
$$

then squaring equation (26) and integrating over the period yields 


$$
\int_{0}^{\tau}\left\{\int_{0}^{t} p(s) d s+\alpha\right\}^{2} d t=\left(\frac{\tau}{2 \pi}\right)^{2} \sum_{r=1}^{\infty} r^{-2}\left(a_{r}^{2}+b_{r}^{2}\right) .
$$

Thus equation (24) has as its dominating equation

where

$$
\ddot{y}+\gamma t^{-2} y=0
$$

$$
\gamma=\frac{1}{2} \int_{0}^{\tau}\left\{\int_{0}^{t} p(s) d s+\alpha\right\}^{2} d t
$$

It may be noted that only weak conditions on the regularity of $p$ are required. Obviously this last case can be extended to treat an equation in which $t^{-k}$, $0<k<1$, replaces $t^{-1}$ in equation 24. Additionally the discussion can be extended to a variety of cases in which the forms considered here are merely the leading terms in an asymptotic expansion of the coefficient of $y$.

\section{Acknowledgement}

Part of this work was done whilst the author was on leave at the Mathematics Research Center, University of Wisconsin. He wishes to acknowledge the hospitality of the Center and support by the United States Army under contract number D.A.-31-124-ARO-D-462.

\section{References}

[1] J. D. Cole, Perturbation Methods in Applied Mathematics (Blaisdell, Waltham, 1968).

[2] N. D. Fowkes, 'A singular perturbation method. Part. I', Quart. App. Maths. 27 (1968) 57-69.

Department of Mathematics

University of Western Australia 